\title{
Is It Useful to Administer Probiotics Together With Proton Pump Inhibitors in Children With Gastroesophageal Reflux?
}

\author{
Oana Belei, ${ }^{1 *}$ Laura Olariu, ${ }^{1}$ Andreea Dobrescu, ${ }^{2}$ Tamara Marcovici, ${ }^{1}$ and Otilia Marginean ${ }^{1}$ \\ ${ }^{1}$ First Pediatric Clinic and ${ }^{2}$ Genetics Department, University of Medicine and Pharmacy Victor Babes, Timisoara, Romania
}

\begin{abstract}
Background/Aims
Gastroesophageal reflux disease (GERD) is a frequent condition diagnosed in children and treated with proton pump inhibitors (PPI). Long-term PPI administration can alter intestinal bacterial population by suppressing the gastric acid barrier and may cause diarrhea. The aim of this study is to evaluate the prevalence of small intestinal bacterial overgrowth assessed by glucose hydrogen breath test among children that received 12 weeks of PPI with or without probiotics (Lactobacillus reuteri DSM 17938) associated, compared to controls.
\end{abstract}

\section{Methods}

Glucose hydrogen breath test was performed before PPI treatment and after 12 weeks of PPI treatment to 128 consecutive children with GERD (1-18 years old) and a control group (120 healthy children). The children with GERD were randomized into 2 groups: placebo group (64 who received PPI and placebo for 12 weeks) and probiotics group (64 who received PPI and probiotics for 12 weeks).

\section{Results}

After 12 weeks of treatment, dysbiosis was detected among $56.2 \%$ of children from placebo group (36/64), compared to $6.2 \%$ of children from the probiotics group $(4 / 64, P<0.001)$. Bacterial overgrowth was detected in $5 \%$ of controls $(6 / 120)$. Probiotics group had a lower prevalence of dysbiosis, similar to controls $(P=0.740)$.

\section{Conclusion}

Probiotics administration decreased the rate of dysbiosis among children treated with PPI.

(J Neurogastroenterol Motil 2018;24:51-57)

\section{Key Words}

Breath tests; Child; Dysbiosis; Probiotics; Proton pump inhibitors

Received: April 24, 2017 Revised: August 12, 2017 Accepted: October 13, 2017

(a) This is an Open Access article distributed under the terms of the Creative Commons Attribution Non-Commercial License (http://creativecommons. org/licenses/by-nc/4.0) which permits unrestricted non-commercial use, distribution, and reproduction in any medium, provided the original work is properly cited.

*Correspondence: Oana Belei, MD, PhD

First Pediatric Clinic, University of Medicine and Pharmacy Victor Babes, No 2, Eftimie Murgu Square, Timisoara 300041, Romania Tel: +40-723289480, Fax: +40-723289480, E-mail: oana22_99@yahoo.com, belei.oana@gmail.com 


\section{Introduction}

In children, gastroesophageal reflux disease (GERD) is considered when the retrograde flow of acid gastric contents into the esophagus induces severe symptoms and/or associated complications such as esophagitis or pulmonary aspiration. ${ }^{1}$ A 4-week trial of antacid secretory agent-histamine $\mathrm{H}_{2}$ receptor antagonists or proton pump inhibitors (PPI) is recommended for pediatric patients who are unable to describe their symptoms (infants, toddlers, young children, and those with neurodisability and communication difficulties) who have overt regurgitations and feeding difficulties, distressed behavior or failure to thrive. ${ }^{1}$ PPI treatment has became the first line therapy prescribed nowadays for children older than one year old and adult patients with GERD.

Several studies have implicated PPI administration in the pathogenesis of small intestinal bacterial overgrowth (SIBO)., ${ }^{2,3}$ Use of PPI could predispose individuals to achieve SIBO by altering the intraluminal environment and bacterial flora. There is controversy regarding the risk of SIBO among PPI users because of conflicting results from prior studies. ${ }^{3,4}$ For several reasons, the composition of the intestinal microbiota can be altered, so that bacterial overgrowth may occur. As the normal upper small bowel harbors less than $10^{4}$ colony forming units (CFU)/mL and the vast majority of these bacteria are Gram positive aerobes, it is possible to define SIBO as any conditions differing from this. ${ }^{5}$ SIBO is usually defined as a total growth of $10^{5} \mathrm{CFU} / \mathrm{mL}$ in intestinal fluid. ${ }^{6,7}$ Affected patients may be asymptomatic or have non-specific symptoms, such as bloating, abdominal pain, diarrhea, steatorrhea, flatulence, dyspepsia, nutrient malabsorption, weight loss, and failure to thrive. $^{8}$

Glucose hydrogen breath test (GHBT) is one of the diagnostic methods used for SIBO detection. ${ }^{5}$ Glucose is a monosaccharide that under normal circumstances is completely absorbed in the small intestine. ${ }^{5}$ In case of bacterial overgrowth in the small intestine, the bacterial fermentation will take place at this level and the process of absorption and fermentation will be competitive. ${ }^{6}$ These are the facts that supported the implementation of GHBT for the diagnosis of SIBO in clinical practice.

The aim of this study is to evaluate the prevalence of SIBO assessed by GHBT among children with GERD that received 12 weeks of antacid secretory agent treatment, compared to controls. We also analyzed the effect of simultaneous probiotic (Lactobacillus reuteri DSM 17938) administration to PPI treatment on reducing the rate of SIBO in children with GERD and monitored the intestinal symptoms in children with GERD treated with PPI and probiotics versus PPI and placebo.

\section{Materials and Methods}

\section{The Basal Characteristics of Subjects}

Between January 2014 and January 2017 the authors conducted a 3-year prospective study at an academic referral pediatric center in the Western part of Romania. GHBT was performed in 248 consecutive children (1-18 years old, mean age $8 \pm 2.2$ years). The inclusion criteria were as follows: 128 consecutive children with GERD treated with PPI for 12 weeks and 120 consecutive healthy age and gender matched subjects. The diagnosis of SIBO in this study was based on a positive GHBT. The development of suggestive symptoms such as abdominal pain/discomfort, bloating, flatulence, diarrhea, weight loss, and/or absence of weight gain was further assessed. The presence of gastrointestinal (GI) symptoms was assessed using a questionnaire with a Likert scale of symptom severity. ${ }^{2}$ The questionnaires were administrated to parents/caregivers of pediatric patients aged below 8 years old and to children themselves in subjects older than 8 years old with optimal cognitive capacity. The questionnaire referred to the GI symptoms over the past 7 days. Each question was rated on a 5-point Likert scale from 0 to 4 . Higher values indicated more severe symptoms. The authors used the Bristol stool scale chart ${ }^{9}$ to assess the stool consistency. The exclusion criteria were as follows: recent gastroenteritis, laxative administration, anti-diarrheal medication, use of antibiotics in the month preceding the study, use of prednisone, drugs that alter intestinal motility, children suffering from diabetes, thyroid disease, pseudo-obstruction, and children who had undergone colonoscopy or enema in the last 4 weeks prior the enrollment.

\section{Classification of the Subjects}

GERD in children was diagnosed based on the North American Society of Pediatric Gastroenterology, Hepatology and Nutrition (NASPGHAN) and European Society of Pediatric Gastroenterology, Hepatology and Nutrition (ESPGHAN) guidelines, ${ }^{10}$ that is mainly based on history and physical examination. Routine laboratory investigations were performed in all cases and only selected cases were referred to upper digestive endoscopy and/or combined esophageal $\mathrm{pH}$ and impedance monitoring. One hundred and twenty-eight children with GERD who received PPI for 12 weeks were consecutively randomized by a hospital based intranet computer system into 2 groups: placebo group (64 who 
received PPI and placebo for 12 weeks) and probiotics group (64 who received PPI and probiotics for 12 weeks). L. reuteri DSM 17938 was administered to the probiotics group. The probiotics group received $5 \mathrm{~mL}$ bottles with odorless and tasteless oral solution. The recommended dose was 5 drops once per day containing $0.1 \times 10^{9} \mathrm{CFU}$. In the placebo group, the children received water bottled in $5 \mathrm{~mL}$ vials with a plastic dropper. The recommended dose was the same: 5 drops once per day. The PPI treatment in children with GERD consisted of esomeprazole $1 \mathrm{mg} / \mathrm{kg}$ daily, once per day (maximum $40 \mathrm{mg}$ ) for 12 weeks. GHBT was performed using LactoFAN analyzer (Fischer ANalysen Instrumente $\mathrm{GmbH}$, Leipzig, Germany) before treatment and after 12 weeks of treatment for every child included into the placebo and probiotics group, and only once at enrollment for controls.

\section{Diagnostic Method of the Glucose Hydrogen Breath Test}

For measuring hydrogen concentrations in breath, the authors used LactoFAN device (Fischer ANalysen Instrumente GmbH, Leipzig, Germany). Before the test, children (except toddlers) were asked to brush their teeth and rinse the mouth with antiseptic mouth wash and tap water, to eliminate an early hydrogen peak due to action of oral bacteria on glucose substrate. After an overnight fast, $1 \mathrm{~g} / \mathrm{kg}$ with a maximum of $50 \mathrm{~g}$ glucose, dissolved in $200 \mathrm{~mL}$ of water was orally administered to each tested child, according to the manufacturer's recommendations. An end-expiratory breath sample was collected immediately before and at every 20 minutes for the next 120 minutes after substrate intake. If the basal value of breath hydrogen was more than $16 \mathrm{ppm}$, it was considered a high value. The glucose was not administered in such situations and the test was rescheduled to be repeated with proper preparation. An increase in breath hydrogen in 2 consecutive measurements of at least $15 \mathrm{ppm}$ above the basal value was considered indicative for SIBO, according to the manufacturer's recommendations.

\section{Statistical Methods}

The statistical analysis was performed using SPSS Statistics for Windows, version 17 (IBM Corp, Armonk, NY, USA). Means and standard deviations were calculated for numerical variables with a normal distribution, whereas for variables with a non-normal distribution, medians and ranges were calculated. Qualitative variables were expressed as numbers and percentages. Chi-test (with Yates' correction for continuity) was used to compare proportions expressed as percentages. For all statistical analyses, a two-tailed $P$ value $<0.05$ was considered significant.

\section{Ethics}

All legal guardians or parents of patients enrolled in this study signed a written informed consent prior to inclusion. Institutional ethical approval with number 1712 was obtained for this study. The work was conducted in compliance with Local Institutional Review Board for Human Subjects Research Committee requirements.

\section{Results}

The demographic data and other characteristics of the study groups (control group, healthy children; placebo group, PPI and placebo for 12 weeks; probiotics group, PPI and probiotics for 12 weeks) are presented in Table. The mean age of basal characteristics was not different among the groups (control group $8.3 \pm 2.7$ years old, placebo group $8.5 \pm 2.4$ years old, probiotics group $7.6 \pm 2.1$ years old). The gender distribution of the patients enrolled in this study was as follows: girls/boys ratio in control group 41/79, in placebo group 29/35 and in probiotics group 21/43. The mean body mass index did not present significant differences among the groups (control group $16.1 \pm 2.1$, placebo group $15.7 \pm 3.0$ and probiotics group $14.3 \pm 3.1$ ). The children with GERD did not associate any comorbidities. Children from placebo and probiotics groups received only the medication prescribed in the study protocol. The children included in the control lot did not receive any drug at the

Table. The Characteristics of the Study Groups

\begin{tabular}{|c|c|c|c|}
\hline Parameters & Control group $(\mathrm{n}=120)$ & Placebo group $(n=64)$ & Probiotics group $(n=64)$ \\
\hline Mean age $\pm \mathrm{SD}(\mathrm{yr})$ & $8.3 \pm 2.7$ & $8.5 \pm 2.4$ & $7.6 \pm 2.1$ \\
\hline Gender (girls/boys) & $41 / 79$ & $29 / 35$ & $21 / 43$ \\
\hline $\operatorname{BMI}\left(\mathrm{kg} / \mathrm{m}^{2}\right)$ & $16.1 \pm 2.1$ & $15.7 \pm 3.0$ & $14.3 \pm 3.1$ \\
\hline Comorbidities (\%) & 0 & 0 & 0 \\
\hline Other drugs intake besides those from study protocol (yes/no) & no & no & no \\
\hline
\end{tabular}

BMI, body mass index. 
time of enrollment. The control subjects did not use PPI, antibiotics or probiotics in the last 4 weeks prior the enrollment (Table).

GHBT was successfully conducted in all enrolled subjects with a few exceptions. Five children with GERD from the placebo group had basal values of hydrogen more than $16 \mathrm{ppm}$. These patients were rescheduled to repeat the GHBT with proper preparation. All 5 children had a lower baseline hydrogen when they came the second time.

The overall frequency of SIBO was $0 \%$ when the GHBT was performed before treatment in both groups of children with GERD: the placebo group and the probiotics group. The preva-

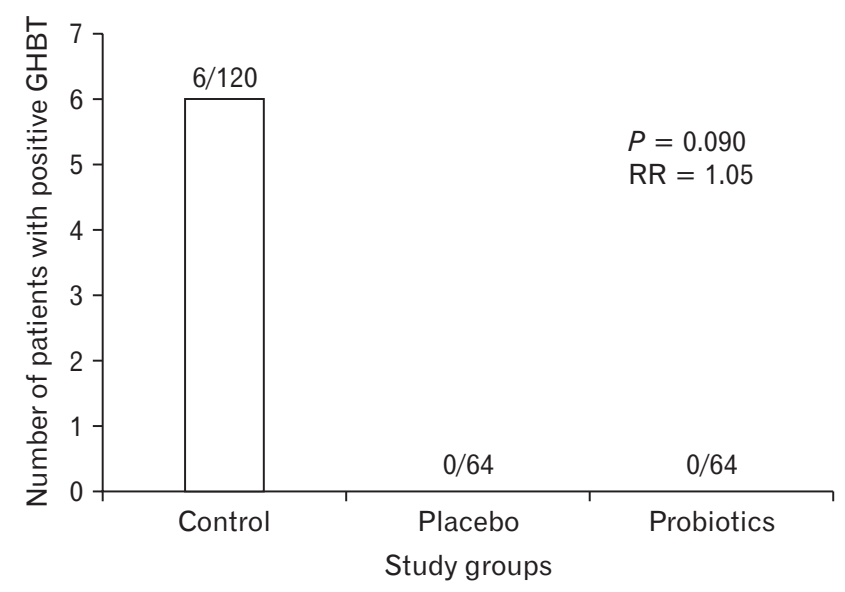

Figure 1. Glucose hydrogen breath test (GHBT) result of control, placebo, and probiotics groups before treatment. RR, risk ratio.

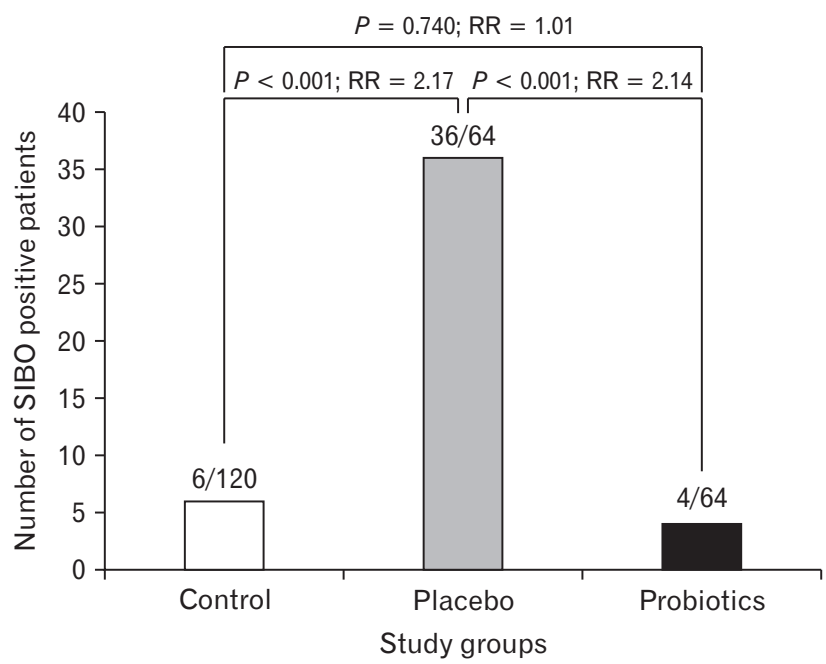

Figure 2. Comparison of small intestinal bacterial overgrowth (SIBO) prevalence as assessed by glucose hydrogen breath test among the study groups. RR, risk ratio. lence of SIBO assessed by GHBT in controls was 5.0\% (6/120; $P$ $=0.090$; risk ratio $[\mathrm{RR}], 1.05 ; 95 \% \mathrm{CI}, 1.01-1.10)$. The results of positive GHBT in the control, placebo, and probiotics groups before treatment are depicted in Figure 1.

After 3 months of continuous PPI administration, SIBO was detected in $36(56.2 \%)$ of the 64 children enrolled in the placebo group, versus $6(5.0 \%)$ of the 120 children in controls $(P<0.001)$, with a RR of $2.17,95 \% \mathrm{CI}, 1.63-2.87)$. The results were statistically significant. Also, there was a significant difference between SIBO prevalence among the placebo group (36 patients [56.2\%]) and the probiotics group (4 patients [6.2\%]) $(P<0.001)$, with a RR of $2.14,95 \%$ confidence interval (CI, 1.61-2.84). Only 4 (6.2\%) of 64 children with GERD treated with simultaneous PPI and probiotics developed a positive GHBT after 12 weeks of treatment. Association of probiotics to esomeprazole significantly decreased the prevalence of SIBO among children with GERD compared to those treated with PPI and placebo $(P<0.001)$. Children with GERD treated with PPI and probiotics had a lower prevalence of SIBO, similar to control lot $(P=0.740)$. Figure 2 depicts the comparison and shows the statistical differences of SIBO prevalence as assessed by GHBT among the control, placebo, and probiotics groups after 3 months of PPI administration.

From the total of 36 children with GERD treated with PPI and placebo with positive GHBT after 12 weeks of treatment, 23 patients (63.8\%) developed intestinal symptoms. These were considered as symptomatic children with SIBO. The rest of the 13 patients $(36.1 \%)$ were free of symptoms, although they all had positive GHBT. All 4 children with GERD from the probiotics group with

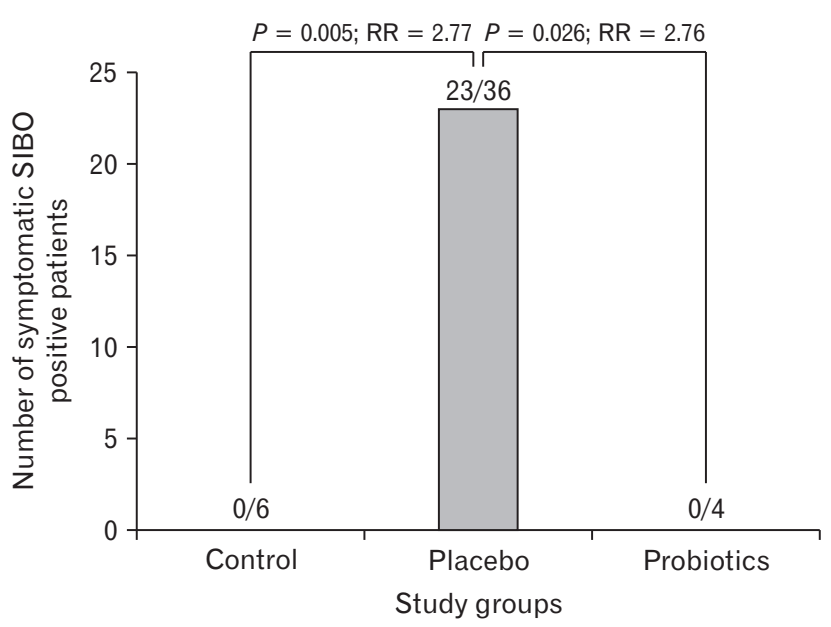

Figure 3. The presence of intestinal symptoms among different groups of children with positive glucose hydrogen breath test. SIBO, small intestinal bacterial overgrowth. RR, risk ratio. 
positive GHBT after 12 weeks of treatment were symptom free. In these cases SIBO was diagnosed based on GHBT positivity only. There were significantly more symptomatic SIBO children among those treated with PPI and placebo compared to the probiotics group $(P=0.026$; RR, 2.76; 95\% CI, 1.79-4.27). All 6 subjects from the control group with positive GHBT were symptom free. Figure 3 shows the distribution of intestinal symptoms among the children with positive glucose hydrogen breath test from the control, placebo, and probiotics groups.

\section{Discussion}

There are several studies reporting different SIBO prevalence among children with different conditions. Siniewicz-Luzeńczyk et $\mathrm{al}^{11}$ reported a prevalence of $63.0 \%$, while a Dutch study conducted by Korterink et $\mathrm{al}^{12}$ reported a lower prevalence of $14.3 \%$ among children with functional abdominal pain. Ojetti et $\mathrm{al}^{13}$ reported the prevalence of SIBO in children with myelomeningocele and constipation as being $39.0 \%$. Wang et $\mathrm{al}^{14}$ found a prevalence of $31.0 \%$ for SIBO among children with autism spectrum disorders. The studies describing the relationship between SIBO and GERD are scarce. This is one of the few studies reporting SIBO prevalence among pediatric patients with GERD. In our study we described a higher prevalence of SIBO among children treated with PPI than previously reported.

Cares et $\mathrm{al}^{15}$ assessed the risk of SIBO with chronic use of PPI in children and he found a potential risk of SIBO in chronic PPI users. However, Cares' results were not statistically significant. SIBO was detected in 5/56 (8.9\%) PPI using group versus $1 / 27$ (3.7\%) control group $(P=0.359)$, with a relative risk of 2.4 in the above cited study. ${ }^{15}$ Sieczkowska et $\mathrm{al}^{16}$ also obtained a lower rate of intestinal dysbiosis $22.5 \%$ of 40 subjects treated for 3 months with PPI), compared to our results. Overall, from 128 children with GERD enrolled in our study and treated for 3 months with PPI (with or without associated probiotics), we found SIBO in 31.2 $\%$ of cases (40/128 children) versus $5.0 \%$ (6/120 controls) $(P<$ $0.001 ; \mathrm{RR}, 1.38$; 95\% CI, 1.22-1.56). Sieczkowska et al ${ }^{16}$ reported that children with intestinal dysbiosis had higher prevalence of GI symptoms than those without SIBO, and the authors concluded that symptomatic patients undergoing PPI treatment should be evaluated for SIBO rather than empirically prolonging antisecretory therapy. A positive GHBT is highly suggestive for diagnosis of SIBO, but it is not always accompanied by the presence on intestinal symptoms. Therefore, the development of suggestive symptoms for SIBO in children with positive GHBT was assessed in our study.
We found a high rate of symptomatic patients with SIBO $(63.8 \%$ 23/36 cases) among children with GERD treated with PPI, strengthening the conclusion of Sieczkowska et $\mathrm{al}^{16}$ that symptomatic children taking PPI treatment should be tested for SIBO.

Hegar et $\mathrm{al}^{17}$ studied the incidence of positive GHBT in a lot of children treated for one month with omeprazole and probiotics Lactobacillus rhamnosus R0011 and Lactobacillus acidophillus R0052 versus a group treated with omeprazole and placebo. There was no difference in the incidence of positive breath tests in the probiotics versus the placebo group $(33.0 \%$ vs $26.5 \% ; P=$ 0.130). ${ }^{17}$ The above authors concluded that the probiotics tested did not decrease the risk to develop SIBO in children treated with omeprazole. ${ }^{17}$ Hegar's results were contradictory compared to our study. We assessed the risk of SIBO after chronic use of esomeprazole in children and demonstrated a potential risk of SIBO when esomeprazole was continuously administrated for at least 3 months. Administration of probiotics $L$. reuteri decreased the risk of intestinal dysbiosis in our study. The frequency of positive GHBT in our study was significantly lower in the probiotics versus the placebo group (6.2\% versus $56.2 \% ; P<0.001)$. The results achieved in our study compared to Hegar's research may be explained by different study design and methodology (longer period of PPI administration - 3 months in our study compared to only 1 month in Hegar's study) and the different pediatric population enrolled.

Rosen et $\mathrm{al}^{18}$ demonstrated that acid-suppression may favor gastric bacterial overgrowth of certain strains including Staphylococcus and Streptococcus. In the above mentioned study, the nonacid reflux was associated with greater concentrations of bacteria in the lung, increasing the risk of upper and lower respiratory tract infections. ${ }^{18}$ Rosen's study had different methodology compared to our study. The authors did not use GHBT. Rosen's research was based on microbiota assessment using cultures from the pulmonary aspirates in patients with GERD treated with PPI that associated aspiration pneumonia. The results converged to a similar conclusion: antisecretory drugs contributed to the change of the individual GI microbiota among patients with GERD. ${ }^{18}$

Lyszkowska et $\mathrm{al}^{19}$ demonstrated that administration of Saccharomyces boulardii for one month alleviated abdominal pain, bloating, flatulence among children with short bowel syndrome. Lyszkowska's study was conducted on a different pediatric population with short bowel syndrome, but the authors have demonstrated that $S$. boulardii induced changes in stool flora and may impact the gut microbiota. Our research focused on another probiotics strain, L. reuteri, and this strain also alleviated digestive symptoms among children with positive GHBT treated with PPI. 
Liang et $\mathrm{al}^{20}$ reported that 116 of 200 patients with GI malignancies (58.0\%) were long-term PPI users and of these, 86 (74.1\%) were positive for SIBO. The authors showed that the group receiving probiotic treatment (Bifidobacterium) and PPI had 19.0\% SIBO-positive patients, whereas the group that was administered placebo and PPI showed $74.6 \%$ SIBO-positive patients. ${ }^{20}$ SIBO prevalence reported by Liang et al ${ }^{20}$ is higher compared to our study both in placebo and probiotics lot (56.2\% and $6.2 \%$ respectively), but this Chinese research was conducted on adults patients with GI malignancies, that might increase the rate of intestinal dysbiosis. The clinical symptoms in the study by Liang et $\mathrm{al}^{20}$ were much diminished in the probiotics group, and this difference reached statistical significance on comparison with the placebo arm of the study $(P<0.05)$. These results are similar to our report. In our study, there were $63.8 \%$ symptomatic SIBO children among those treated with PPI and placebo while in the probiotics group none of the 4 children with positive GHBT presented intestinal symptoms $(P=$ 0.026). It could be a beneficial effect of probiotics association.

Other papers suggested that probiotics may enhance the efficiency of antibiotics for SIBO eradication. ${ }^{21}$ One study showed that treatment with rifaximin along with probiotic Lactobacillus casei improved the symptoms of SIBO more effectively than the antibiotic followed by probiotics. ${ }^{21}$ Our research sustained the beneficial effect of $L$. reuteri in preventing intestinal bacterial overgrowth among children treated with PPI. Based on the results of our study, in order to decrease the rate of SIBO, we recommend administrating probiotics based on $L$. reuteri to all children with GERD treated with long-term antisecretory medication. More studies, however, are needed to evaluate the efficacy of probiotics among children treated with PPI in relation to occurrence of SIBO.

Nowadays, hydrogen breath tests, using glucose or lactulose as substrates are the most common diagnostic tools for SIBO. Hydrogen breath tests with lactulose may be able to diagnose only onethird of patients with SIBO according to some authors. ${ }^{22,23}$ Kerlin and Wong ${ }^{24}$ have reported that GHBT performed for 2 hours had a sensitivity of $93.0 \%$ and a specificity of $78.0 \%$ in SIBO identification against the gold standard of a jejunal aspirate. Therefore, in this study the authors assessed SIBO by using glucose as the substrate for hydrogen breath tests. GHBT in our study identified all subjects with SIBO, except methane producing bacteria overgrowth.

There are some pitfalls in the interpretation of GHBT: since glucose is absorbed completely in the upper small intestine, it may not be able to diagnose SIBO of the distal small intestine. ${ }^{22}$ Some studies described a considerable number of non-hydrogen producing bacteria, that produce other gases such as methane and hydrogen-sulfide and may not be detected with the hydrogen breath test, inducing false negative results for SIBO ${ }^{5,22}$ The discrepancy between GHBT positivity and presence of symptoms found in this study could also be explained by other sources of error. Delayed gastric emptying may cause false negative results, and rapid transit through the small bowel may produce false positive hydrogen breath tests. False positive results may also be due to the oral bacterial flora and in cases of non-compliance to follow to a low fiber diet 24 hours prior to the test. ${ }^{5}$ Based on these observations, we may speculate that in our study some children with positive GHBT without any GI symptoms had false positive breath test results due to rapid small bowel transit. Oral flora contamination could not be a confounding factor for false positive results in our study because we have ensured rigorous oral hygiene in all children prior to inclusion, and all subjects followed a low fiber diet 24 hours prior to the test.

A limitation of this study is represented by the fact that we did not assess the methane concentration in exhaled breath air. Therefore, we may have missed the identification of certain non-hydrogen producing bacteria overgrowth. Another limitation of this study is the fact that we did not use jejunal cultures for SIBO assessment. Culture of the jejunal aspirate is recognized as the most direct method for diagnosing SIBO. ${ }^{4}$ Yet, obtaining jejunal aspirates implies invasive procedures hardly accepted by parents in pediatric patients. In children with isolated distal SIBO, it could remain undiagnosed despite using jejunal cultures. ${ }^{25}$ Due to all these disadvantages of jejunal aspirates, GHBT was used in this study as an indirect, but reliable alternative test to assess SIBO. ${ }^{4,5,22}$

In conclusion, the administration of probiotics not only decreased the rate of SIBO among children with GERD treated with PPI, but also significantly reduced the expression of digestive symptoms encountered in the group with positive GHBT. Being a disorder that requests long-term antisecretory therapy, GERD may benefit by acid suppression combined with probiotics in order to decrease the risk of intestinal bacterial alteration. Routine administration of the strain $L$. reuteri could be beneficial to all children with GERD treated with PPI in order to prevent SIBO. Nevertheless, PPI long-term administration in children should be avoided and "step-down" or "on demand" strategy should be implemented.

\section{Financial support: None.}

\section{Conflicts of interest: None.}

Author contributions: Oana Belei conducted the study, contributed to the conception and the design of the study, to the 
collection, analysis, and interpretation of data, and revised and approved the final version to be published; Laura Olariu contributed to the collection, analysis, and interpretation of data, and approved the final version to be published; Andreea Dobrescu drafted the manuscript, contributed to the collection, analysis, and interpretation of data, and approved the final version to be published; Tamara Marcovici contributed to the collection, analysis, and interpretation of data, and approved the final version to be published; and Otilia Marginean contributed to the collection, analysis, and interpretation of data, and approved the final version to be published.

\section{References}

1. Davies I, Burman-Roy S, Murphy MS; Guideline Development Group. Gastro-oesophageal reflux disease in children: NICE guidance. BMJ 2015;350:g7703.

2. Jacobs C, Coss-Adame E, Attaluri A, Valestin J, Rao SSC. Dysmotility and PPI use are independent risk factors for small intestinal bacterial and/ or fungal overgrowth. Aliment Pharmacol Ther 2013;37:1103-1111.

3. Lo WK, Chan WW. Proton pump inhibitor use and the risk of small intestinal bacterial overgrowth: a meta-analysis. Clin Gastroenterol Hepatol 2013;11:483-490.

4. Chandra S, Dutta U, Noor MT, et al. Endoscopic jejunal biopsy culture: a simple and effective method to study jejunal microflora. Indian J Gastroenterol 2010;29:226-230.

5. Simren M, Stotzer PO. Use and abuse of hydrogen breath tests. Gut 2006;55:297-303.

6. Rumessen JJ, Gudmand-Høyer E, Bachmann E, Justesen T. Diagnosis of bacterial overgrowth of the small intestine. Comparison of the ${ }^{14} \mathrm{C}-\mathrm{D}$ xylose breath test and jejunal cultures in 60 patients. Scand J Gastroenterol 1985;20:1267-1275.

7. Donald IP, Kitchingmam G, Donald F, Kupfer RM. The diagnosis of small bowel bacterial overgrowth in elderly patients. J Am Geriatr Soc 1992;40:692-696.

8. King T. Small intestinal bacterial overgrowth and irritable bowel syndrome. JAMA 2004;292: 2213; author reply 2213-2214.

9. Lewis SJ, Heaton KW. Stool form scale as a useful guide to intestinal transit time. Scand J Gastroenterol 1997;32:920-924.

10. Vandenplas Y, Colin DR, Di Lorenzo C, et al. Pediatric gastroesophageal reflux clinical practice guidelines: joint recommendations of the North American Society of Pediatric Gastroenterology, Hepatology, and Nutrition (NASPGHAN) and the European Society of Pediatric Gastroenterology, Hepatology and Nutrition (ESPGHAN). J Pediatr Gastroenterol Nutr 2009;49:498-547.

11. Siniewicz-Luzeńczyk K, Bik-Gawin A, Zeman K, Bąk-Romaniszyn L.
Small intestinal bacterial overgrowth syndrome in children. Prz Gastroenterol 2015;10:28-32.

12. Korterink JJ, Benninga MA, van Wering HM, Deckers-Kocken JM. Glucose hydrogen breath test for small intestinal bacterial overgrowth in children with abdominal pain-related functional gastrointestinal disorders. J Pediatr Gastroenterol Nutr 2015;60:498-502.

13. Ojetti V, Bruno G, Paolucci V, et al. The prevalence of small intestinal bacterial overgrowth and methane production in patients with myelomeningocele and constipation. Spinal Cord 2014;52:61-64.

14. Wang L, Yu YM, Zhang YQ, Zhang J, Lu N, Liu N. Hydrogen breath test to detect small intestinal bacterial overgrowth: a prevalence casecontrol study in autism. Eur Child Adolesc Psychiatr Published Online First: 10 Aug 2017. doi: 10.1007/s00787-017-1039-2.

15. Cares K, Al-Ansari N, Macha S, et al. Short article: risk of small intestinal bacterial overgrowth with chronic use of proton pump inhibitors in children. Eur J Gastroenterol Hepatol 2017;29:396-399.

16. Sieczkowska A, Landowski P, Zagozdzon, Kaminska B, Lifschitz C. Bacterial overgrowth associated with persistence of abdominal symptoms in children treated with proton pump inhibitors. J Pediatr 2015;166:13101312, e1.

17. Hegar B, Hutapea E, Advani N, Vandenplas Y. A double-blind placebo-controlled randomized trial on probiotics in small bowel bacterial overgrowth in children treated with omeprazole. J Pediatr (Rio J) 2013;89:381-387.

18. Rosen R, Amirault J, Liu H, et al. Changes in gastric and lung micromicrobiota with acid suppression: acid suppression and bacterial growth. JAMA Pediatr 2014;168:932-937.

19. Łyszkowska M, Popińska K, Idzik M, Książyk J. Probiotics in children with gut failure [abstract]. J Pediatr Gastroenterol Nutr 2008;46:543.

20. Liang S, Xu L, Zhang D, Wu Z. Effect of probiotics on small intestinal bacterial overgrowth in patients with gastric and colorectal cancer. Turk J Gastroenterol 2016;27:227-232.

21. Rosania R, Giorgio F, Principi M, et al. Effect of probiotic or prebiotic supplementation on antibiotic therapy in the small intestinal bacterial overgrowth: a comparative evaluation. Curr Clin Pharmacol 2013;8:169172.

22. Ghoshal UC. How to interpret hydrogen breath test. J Neurogastroenterol Motil 2011;17:312-317.

23. Ghoshal UC, Ghoshal U, Das K, Misra A. Utility of hydrogen breath tests in diagnosis of small intestinal bacterial overgrowth in malabsorption syndrome and its relationship with oro-cecal transit time. Indian J Gastroenterol 2006;25:6-10.

24. Kerlin P, Wong L. Breath hydrogen testing in bacterial overgrowth of the small intestine. Gastroenterology 1988;95:982-988.

25. Shimura S, Ishimura N, Mikami H, et al. Small intestinal bacterial overgrowth in patients with refractory functional gastrointestinal disorders. J Neurogastroenterol Motil 2016;22:60-68. 